\title{
A COMPOSITE CONTROL STRATEGY FOR SENSORLESS AND LOW-NOISE OPERATION OF SWITCHED RELUCTANCE MOTOR DRIVE
}

\author{
Debiprasad Panda, Student member IEEE, and V. Ramanarayanan \\ Department of Electrical Engineering, \\ Indian Institute of Science, Bangalore 560012, India \\ email:dpanda@ieee.org. vram@ee.iisc.ernet.in
}

\begin{abstract}
In this paper, a composite control strategy for sensorless and low-noise operation of Switched Reluctance (SR) is proposed. In the proposed method dc bus voltage is varied as a function of speed and the motor is operated in single pulse mode for wide speed range. Such controller reduces the acoustic noise and improves the accuracy of estimation. The algorithm is tested on a 4-phase $8 / 6$ pole, $4 \mathrm{~kW}$ SR motor and the test results of acoustic noise, position estimation are presented.
\end{abstract}

Keyword: Sensorless, Low-Noise, Position estimation, Acoustic noise, Radial force.

\section{INTRODUCTION}

The SR motor is a promising candidate for various adjustable speed drives in industrial and consumer product applications. However, the rotor position sensing requirement and higher acoustic noise are major disadvantages for this motor. The motivation of the present work is to develop a composite controller which will reduce the acoustic noise of the motor and improve the accuracy of estimated position. In this paper, a control strategy based on variable dc bus voltage is proposed. In order to realise this algorithm, a front end chopper is used for controlling the $\mathrm{dc}$ bus voltage and the conventional split-link capacitor converter is used for controlling the dwell angle of the phases of the motor. With such converter, the dc bus voltage is controlled as a function of speed. The reduced voltage operation at low speeds reduces the acoustic noise. Besides, it improves the accuracy of estimated position by reducing the error in computed flux due to sampling , qunatisation and integration. A DSP based control platform is used for implementation. The proposed method is tested on an $8 / 6,4-\mathrm{kW}$ SR motor and the relevant test results of sensorless operation and noise reduction are presented.

II SOURCES OF ACOUSTIC NOISE IN SR MOTOR AND EXISTING REMEDIES

Despite a number of positive attributes, the SR motor drive exhibits higher level of acoustic noise and vibration than most competing drives. Hence, attention must be given on integrating acoustic noise considerations into the overall control aspects of SR motor drive. It is only in recent years that serious attempts have been made to analyse its acoustic noise and to reduce it to an acceptable level. The first systematic study of SR motor noise was published by Cameroon et al [1, 2]. They used a series of carefully designed experiments to study the different electromagnetic and mechanical sources of noise in SR motor. The possible noise sources in SR motor, as reported by them are discussed below.

\section{A. Radial Force}

There is a strong radial magnetic attraction between the stator and the rotor of the SR motor because of the doubly salient structure. The stator vibration is found to be proportional to the rate of demagnetisation. During commutation, when the phase winding is demagnetised, the stator vibrates, and this vibration produces high acoustic noise. The radial force $F \boldsymbol{r}$ [1] is expressed by

$$
F_{r}(\theta, \lg , t)=-\frac{1}{2} i(t)^{2} \frac{L(\theta(t), i(t), \lg )}{l_{g}}
$$

where $\theta$ is the rotor position, $l g$ is the minimum airgap between the stator and the rotor at the aligned position, $\mathrm{L}$ is the self-inductance of the winding, and $i$ is the winding current.

\section{B. Torque ripple}

Under conventional control algorithms, the SR motor exhibits considerable torque ripple which is a potential source of noise.

\section{Winding vibration}

Winding vibration produced by the interaction of current in the stator windings with leakage flux $(I \times B$ - Lorentz Force) is also a source of noise.

\section{Compression under magnetic effect (Permeance variation)}

Magnetostrictive forces are present in all compressible magnetic materials under the influence of a magnetic field. These forces are generated by the tendency of the magnetic circuit to adopt a configuration of minimum energy. These forces compress the material and thus increase its effective permeance. The vibration in all parts of the magnetic material caused by this force produces noise.

\section{E. Mechanical imperfections}

Faulty bearings and other mechanical defects lead to noisy vibration.

Each of the above sources are studied by Cameron et al $[1,2]$ and through a process of elimination, they have verified experimentally that the radial attraction of the stator and the rotor is the dominant source of acoustic noise in the doubly salient SR motors.

It is verified by Cameron et al that the ovalisation or modal deflection of the stator back-iron as a ring, and the lateral rocking of the stator poles are main sources of noise in SR motor. The frequency domain analysis was used to 
show that the acoustic noise radiated from the machine had significant components which correspond to the natural resonant frequency of the stator. Their study pointed out the importance of resonant vibratory modes. Later on $\mathrm{Wu}$ and Pollock [3] studied the behaviour of the vibration and radial force in time domain. The study was carried both in simulation and experiment. Their study reveals that the radial force reaches its peak at the point of commutation, and as the voltage reverses, it starts decreasing rapidly. This sharp change in gradient of the radial force initiates the radial vibration of the stator along the axis of the stator pole. In their analysis, the radial acceleration of the stator was obtained by introducing a radial force on a second order mechanical model and the direction of the initial acceleration was found negative. In their study, the radial vibration was modelled through finite element method (FEM).

\section{EXISTING NOISE REDUCTION TECHNIQUES IN SR MOTOR}

Acoustic noise in SR motor may be reduced following two approaches- (1) through electromagnetic and mechanical design of the motor; and (2) through proper control algorithm.

The acoustic noise produced by the SR motor becomes prominent when the frequency of vibration coincides with the natural frequency of the stator. Hence, it is important to find out the vibrations modes of the stator. Colby et al [4] have examined the natural modes and frequencies of a 4-phase, 8/6 SR motor. Structural FEM is used to compute the natural modes and frequencies. An approximate formula is derived by them to predict the frequency of the fundamental vibration mode in terms of lamination dimensions and material properties. The formula is validated by comparison with FEM calculations for several laminations, and hence is shown to be useful in the design trade-off studies.

Besbes et al [5] carried out a parametric study to quantify the influence of the stator geometry upon vibratory mode of a $6 / 4$ SR motor. Their study shows the important influence of stator yoke thickness on vibration reduction compared to other stator shape parameters. Finally, they proposed a less noisy structure based on the simultaneous analysis of electromagnetic performances and elastic characteristics.

J. Mahn et al [6] have proposed an approach to investigate the noise and vibration of SR motors. The experimental and analytical approaches used in the study of vibration and noise is discussed in details in their paper. The step by step mechanical and electromagnetic modelling techniques are introduced and some preliminary results of their study are given in that paper.

The above findings mentioned by different authors may be used in the design stage of the motor and proper design algorithm may be devised for reducing the noise in SR motor. Alternatively, by adopting proper control algorithm the noise level may be reduced. The different existing control techniques for noise reduction is explained below.

\section{A. Two-stage Commutation method}

$\mathrm{Wu}$ and Pollock proposed a counter excitation principle $[3,7]$ for cancelling the vibration. In their method, they have adopted a two stage controlled commutation. As mentioned earlier, step change in voltage gradient at the point of commutation initiates a vibration in the stator and that vibration produces the noise. The two-stage commutation method uses two successive steps to reverse the voltage across the phase winding. In the first step, the voltage is reduced from positive to zero producing the first vibration, with initial negative acceleration. After half of a resonant cycle of the stator vibration, the voltage is changed from zero to negative, producing a second vibration at $180^{\circ}$ out of phase with the first, resulting in substantial cancellation of the vibration. In this method, significant vibration persists only for a half a cycle. Later on, Michaelides and Pollock [8] developed an advanced two-stage method through which a more appropriate noise cancellation is obtained.

For successful implementation of this noise cancellation method an accurate modelling of the vibration is required. In case of an error in the model, this method may become counter productive in the sense that the vibrations due to successive voltage variation may add to each other instead of cancelling.

\section{B. Three stage commutation}

The three stage commutation is same in principle as that of two stage commutation. The converters with single switch per phase do not provide the zero voltage loop. Hence two stage method can not be used with such configuration. A Three stage method of active noise cancellation is proposed for these configurations by Pollock et al [7]. This method also suffers from the same disadvantage as that of two-stage method.

\section{Voltage smoothing method}

An alternative commutation strategy was developed by Pollock et al [7] for smoothening the rate of change of applied voltage at the point of commutation. If the voltage is decreased gradually, the rate of change of radial force will be smoothened and vibration will be reduced. In order to achieve smooth reversal of voltage, a period of pulse width modulation (PWM) was applied, where the duty cycle was decreased gradually from its initial value to zero. In this process the vibration is reduced. It is concluded by the above authors that in this process, the switching frequency must be greatly in excess of the resonant frequency of the stator. This high frequency switching (more than $20 \mathrm{kHz}$ ) may not be feasible at all power level. Besides, the duty cycle of the PWM has to be changed continuously within a short span of time, which introduces more complexity in the controller.

\section{Other methods}

The noise may be reduced by selecting the T-off angle away from the aligned position [3]. There are two 


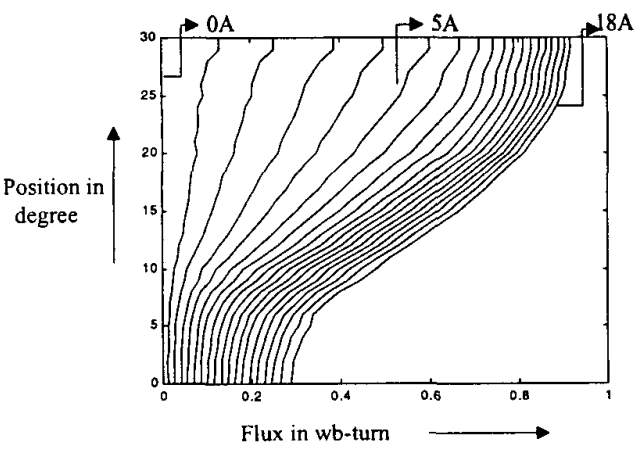

Fig. 1 Flux linkage Characteristics of a $4 \mathrm{kw} 8 / 6$ SR motor

disadvantages to this method. The motor efficiency is reduced and it limits the flexibility in the choice of current commutation. Again by introducing dither in T-on and T-off angles the noise may be reduced [2]. This method has been demonstrated at certain operating points and found effective. But the noise behaviour throughout the operating region is not studied.

Hence, it may be concluded that all the above methods have limitations. Either they operate only at certain operating conditions or they need accurate modelling of the vibrations for noise cancellation.

In this work, an alternative control strategy based on dc bus voltage control is discussed. The control strategy, its implementation and the experimental results of acoustic noise are presented.

\section{Position Estimation}

Rotor position information is essential for driving an SR motor. Normally absolute encoders are used for obtaining the rotor position. It is well discussed that these position sensors may cause reliability problem to the drive and they occupy extra space and add to the total cost of the drive. The researchers are aiming to avoid the position sensors by estimating the rotor position through terminal measurements. A lot of estimation algorithms are suggested for sensorless operation of SR motor $[9,10]$. Most of the position estimation algorithm makes use of the flux-linkage characteristics of the motor for deriving the dynamic position. Such position estimation algorithms are called the flux-current method [11]. In this method, the flux-linkage characteristics (c.f. Fig. 1) of the machine is stored in the form of position as a function of flux and current $(\theta=f(\Psi, i)$ ). The current and voltage of an active phase are measured and flux-linkage is computed with Eq.(5).

$$
\Psi=\int(v-i R) d t
$$

With known flux and current, position is looked up from the stored characteristics. Normally, this kind of estimator is used with conventional controller of low speed chopping control and high speed angle control. In literature, it is reported that at low-speed and with chopping control the accuracy of estimated position is poor on account of error due to sampling and quantisation, resistance variation, sensor drift etc.

\section{A. Sensor drift and measurement error}

In low speed, the integration in Eq.(2) is conducted over relatively longer time. Any drift or error in measured voltage and current will be integrated for the whole time period. For obvious reason, such anomaly will cause large error in estimation of flux which in turn will affect the accuracy of estimated position.

\section{B. Sampling and quantisation error}

The voltage equation of the SR motor winding may be expressed in the form of following equation.

$$
i=\frac{1}{R}\left(v-l \frac{d i}{d t}-e_{b}\right)
$$

In low speed operation, the back emf $e_{b}$, is smaller compared to the supply voltage $v$. In such case, the rate of rise and fall of current is predominantly decided by the supply voltage and the incremental inductance $l$, of the winding. With conventional control method, the dc bus is maintained to the rated voltage of the motor irrespective of speed. Thus at low speed, the rate of change of current will be faster compared to high speed operation. For digital implementation, the current and voltage are sampled at regular sampling interval $\left(T_{S}\right)$ and assuming that the above quantities remain constant over a sampling interval, the flux is computed using Eq. (2). Since, the rate of change of current is faster in low speed, the current varies considerably within one sample period. Such variation of current violates the above assumption and in turn introduces an error in computed flux. In our test machine ( $4 \mathrm{~kW}, 8 / 6$ OULTON motor) the incremental inductance varies form $10 \mathrm{mH}$ to $110 \mathrm{mH}$ and the rated phase voltage is $280 \mathrm{~V}$. If we neglect the resistance drop, then the variation of current within one sample (typically $100 \mu s$ ) period may be calculated as given below.

$$
\begin{aligned}
i & =\frac{v}{l} * \Delta t=\frac{280}{10^{-3}} * 100 * 10^{-6} \\
& =2.8 \mathrm{~A}
\end{aligned}
$$

With such variation of current, the computed flux will be erroneous on account of anomaly in resistance drop. Besides, the integration time being larger in low speed, this error will be accumulated over longer duration. Thus the error in computed flux will be high enough to influence the estimated position. This error may be reduced either by faster sampling or by minimising the current variation between two samples. The sampling time is decided by the software overhead of the processor. By using more expensive processor, faster sampling may be achieved. In this work an alternative controller based on variable $\mathrm{dc}$ link voltage is adopted which reduces the change of current between two samples inherently.

\section{Resistance variation}

During dynamic operation, the temperature of the winding varies and that causes the variation of winding 


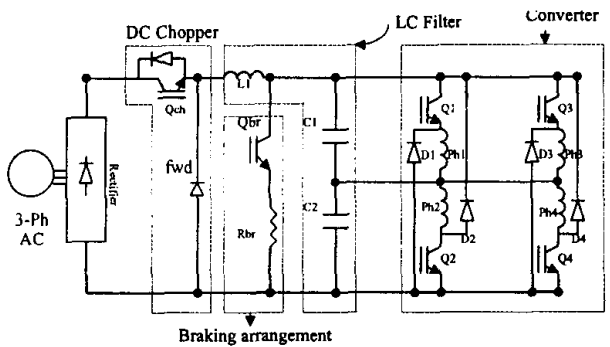

Fig. 2 Power Converter with DC link chopper

resistance. If this variation is not taken into account, the computed flux will be erroneous. In low speed chopping control, this effect is severe. With chopping due to repeated reversal of voltage across the phase, the voltage in the positive and negative half are largely self cancelling, whereas the $i R$ drop remains unidirectional for both the half. In such case, the resistance variation may effect the estimated position considerably. The effect of resistance can be explained more clearly with the following equation. The flux-linkage during on-time, off-time and total flux-linakge over a complete chopping is expressed in the foilowing Eqns.

$$
\begin{aligned}
& \Delta \Psi_{\text {on }}=(v-i R) * T_{\text {on }} \\
& \Delta \Psi_{\text {off }}=(-v-i R) * \text { Toff } \\
& \Delta \Psi=\Delta \Psi_{o n}+\Delta \Psi_{o f f} \\
& =v *\left(T_{o n}-T_{o f f}\right)-i R\left(T_{o n}+T_{o f f}\right)(5 \mathrm{c})
\end{aligned}
$$

From these expressions it may be seen that the voltage is multiplied by the difference of on-time and off-time, whereas, $i R$ is multiplied by the sum of them. Thus the effect of resistance variation will be predominant in chopping mode of operation. In high speed and with single pulse operation, the voltage being much larger than resistance drop and voltage being unidirectional the resistance variation effect is negligible.

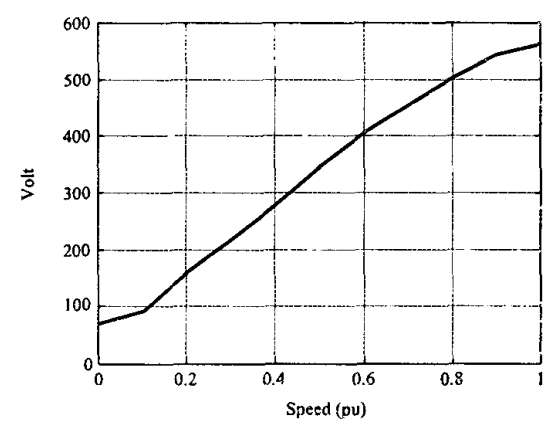

Fig. 3 Voltage (dc bus voltage) vs Speed
From the above discussion, it may be concluded that the sensor error, sampling and quantisation error and the resistance variation effect are to be minimised in order to obtain more accurate estimate of position. Apart from these, mutual flux due to other conducting phase and eddy current in the coupled conducting paths also will influence the estimated position. The effect of these factors on estimation are discussed in separate publication $[12,13]$.

\section{COMPOSITE CONTROL FOR LOW-NOISE AND SENSORLESS} OPERATION OF SR MOTOR

It is discussed earlier that the radial vibration is the major source of noise in SR motor. The noise peaks when the harmonics of the radial force and resonance frequencies of the stator coincide. As explained earlier, it is the rate of withdrawal of radial force that decides the level of noise. Hence, a gradual release of the force should reduce the noise. From (1), it is clear that radial force $F r$ is a function of retor position as well as $i^{2}$. As the position of the rotor gets closer to the aligned region during commutation, the noise increases. Similarly, if the current in the winding is extinguished instantaneously by applying a higher negative voltage across the phase, the noise will be very high. The implication is that noise can be brought down considerably by controlling the current gradient. The current gradient can be made gradual by making the dc bus voltage a function of speed. Thus the dc bus voltage control method is a desirable and feasible option for noise abatement in an SR motor.

\section{A. DC bus voltage control}

The SR motor can be subjected to either constant dc bus voltage control or variable dc bus voltage control [14]. In the conventional control, dc bus voltage is maintained to its rated value irrespective of speed. Currents of individual phases are controiled by hysteresis controi or by constant frequency chopping in the low speed region and conduction angle control in the high speed region $[14,15]$. In this conventional method, full voltage is applied across the winding, resulting in large current gradients. This higher gradient in current incieases the acoustic noise.

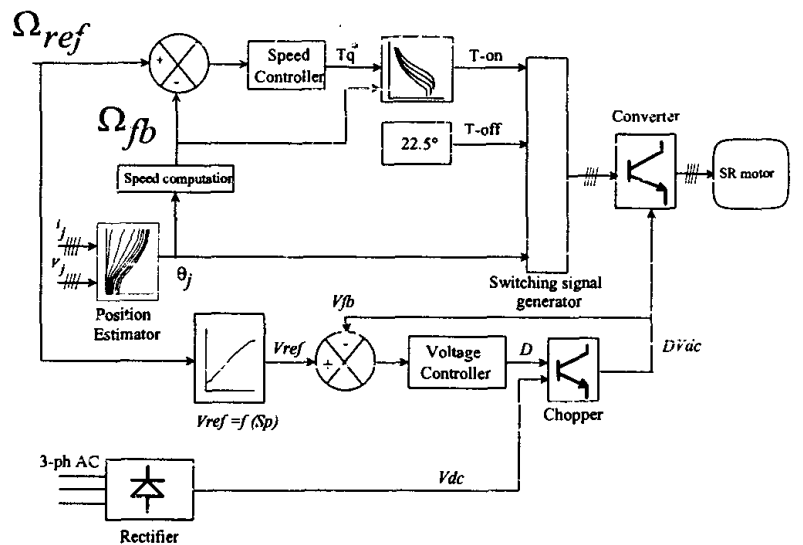

Fig. 4 Cintroller block diagram with Estimator 
In the method proposed in this chapter, a chopper circuit is incorporated between the rectifier and the power converter of the SR motor. The complete power converter for the proposed method is given in Fig. 2. With this power converter, the motor may be supplied with variable voltage. This variable voltage is made a function of speed so that it is capable of maintaining sufficient current in the presence of the back emf and operates in single pulse mode. In such a method, the current gradient in the windings during commutation is substantially reduced. Thus voltage control method reduces the noise to a level considerably lower value than in fixed voltage control.

Further, the machine is controlled in single-pulse mode down to very low speed, which makes it eminently suited to sensorless operation. It is discussed earlier that during low speed operation quantisation error may creep in due to large change in current between two samples when full voltage is applied across the winding. With the proposed method, with lower supply voltage at low speed and with single pulse type of operation the rate of change of current is reduced considerably. The proposed control scheme is thus capable of giving higher-accuracy information of position. A function generator of dc bus voltage vs speed is obtained through simulation for realising such controller. The voltage vs speed plot is given in Fig. 3.

VI. REALISATION OF THE COMPOSITE CONTROL ALGORITHM

A TMS320c5x-based DSP-board is used for the implementation of the algorithm proposed here. A block diagram of the complete controller is shown in Fig. 4. The $\mathrm{dc}$ bus voltages and the phase currents are fed to the DSP through a multi-channel ADC with simultaneous sample and hold. These signals are processed by the DSP, and the DSP provides five switching signals, one for each of the four phases of the motor, and one switching signal for the dc chopper switch control through a digital I/O port. The total control including position and speed estimation, execution of speed controller, selection of appropriate T-on angles, voltage reference selection and control of chopper duty cycle etc. are all carried out by the DSP.

The commanded speed $\Omega_{r e f}$ is compared with the actual speed $\Omega_{f b}$; the difference between these two is the speed error. This error is fed to a PI controller. The output of the $\mathrm{PI}$ controller determines the T-on angle. The T-on angle is varied from $-5.5^{\circ}$ to $+15^{\circ}$ depending on the demand of load and speed. On the other hand, the T-off angle is kept fixed at $22.5^{\circ}$ irrespective of speed and load. The dc bus voltage is made a function of speed. Depending on the reference speed, the reference voltage is varied between $15 \%$ and $100 \%$ of the supply voltage. During starting, dc bus voltage is kept at $80 \mathrm{~V}$. A $415 \mathrm{~V}, 3$-phase AC supply is fed to a rectifier bridge and dc bus voltage is controlled using a chopper between the rectifier and the split-capacitor converter. An L-C filter is used for smoothening the dc bus voltage. The full voltage across the $\mathrm{dc}$ bus is $560 \mathrm{~V}$. The duty ratio of the chopper circuit is controlled using another PI controller. DC bus voltage is used as feedback for this purpose. The machine is loaded through a dc machine coupled to its shaft.

\section{Results AND Discussions}

The control strategy with variable dc link voltage is verified in simulation and experiment. First the performance of the system configured according to the above scheme is studied through simulation using SIMULINK. The speed to voltage function generator is verified in simulation and it is found that the relationship given in Fig. 3 works well for all the operating points. A satisfactory controller is obtained for the speed and voltage controller through simulation. The same controller is implemented on DSP and performance of the controller is tested on a real motor. The motor is run both with and without position sensors and the performance is studied and compared with the simulation results. The acoustic noise with the proposed controller is measured and compared with a commercially available controller. The accuracy of estimated position is tested and compared with a known position sensor output. The steady-state and transient performance of the controller is verified and that test results are presented. The detail simulation and experimental results are discussed in the following sections.

\section{A. Steady-state and Transient Performance}

The simulation results of steady-state phase current and phase voltages for various operating conditions are given in Figs. 5a to 8a. The same control strategy is implemented using DSP and tested on a real motor $(8 / 6$ pole $4 \mathrm{~kW}$ SR motor). The test results with position sensors for the same operating points are presented in Figs $5 \mathrm{~b}$ to $8 \mathrm{~b}$. The results without position sensors are given in Figs $5 \mathrm{c}$ to $8 \mathrm{c}$. From the above figures, a comparative study of the simulation results and test results with and without position sensors may be made. Figure 9 shows the transient waveforms of speed following the above control strategy when the motor was running without position sensors.

\section{B. Acoustic noise reduction}

The acoustic noise is measured with a Bruel \& Kjaer 2235 Precision Sound Level Meter. The sound meter is kept 1 metre away from the motor axis in the radial direction. First the sound level is measured at different speeds of the $\mathrm{DC}$ motor. Then the SR motor is driven by a commercial drive controller (OULTON motor and controller manufactured by M/s Tasc Drive of UK) and the noise level is measured at different speeds (Experiment A). Then SR motor is driven with our DSP based fixed dc bus voltage conventional control method and noise level is measured at different speed (Experiment B). Finally the SR motor is driven by the proposed dc bus voltage controller and the sound level is measured at different speeds (Experiment C). The environmental noise during the experiment is noted separately $(60 \mathrm{~dB})$. The additional noise caused by the DC motor is subtracted from the noises of Experiments A, B and $C$. The remainder figures can be taken as the noises due to the SR motor. The experiments are repeated for different loads $(25 \%, 50 \%, 75 \%$ and $100 \%$ load). It is not the case always that the noise level is at its highest under full-load 
(a)

(i)

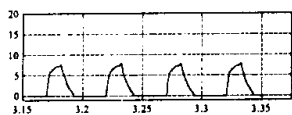

(ii) $\stackrel{\frac{n}{c}}{=}$

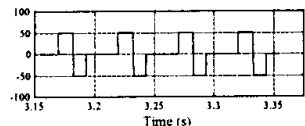

(b)

(i)

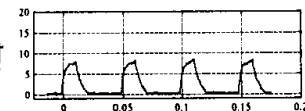

(ii)

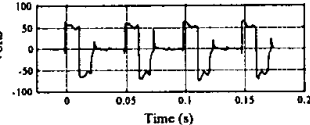

(c)

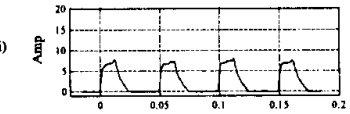

(ii)

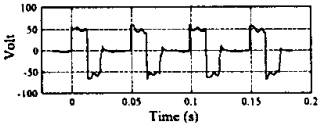

Fig. 5 (i) Phase current and (ii) phase voltages: (a) Simulation results, (b) Experimental result with position sensor and (c) Experimental results without position sensor.

$[\mathrm{Vph}=55 \mathrm{~V}$, Speed $=200 \mathrm{rpm}$, Load $=6.5 \mathrm{Nm}$. $]$

(a)

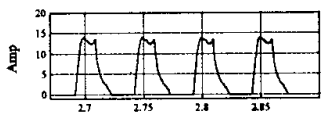

(ii)

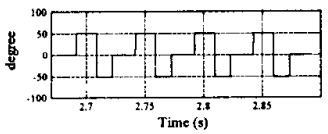

(i)

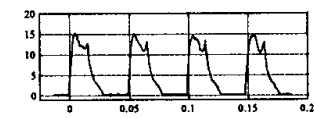

(i) $\frac{\overrightarrow{5}}{3}$

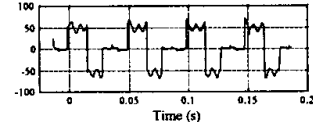

(c)
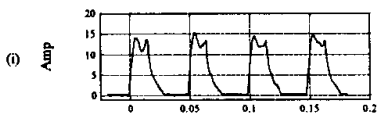

(ii)

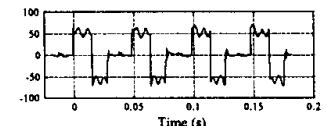

Fig. 6 (i) Phase current and (ii) phase voltages: (a) Simulation results, (b) Experimental result with position sensor and (c) Experimental results without position sensor.

$[\mathrm{Vph}=55 \mathrm{~V}$, Speed $=200 \mathrm{rpm}$, Load $=20 \mathrm{Nm}$.

(a)

(i)

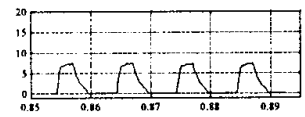

(ii) $\overrightarrow{\overrightarrow{0}}$

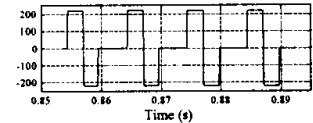

(b)

(i) 量

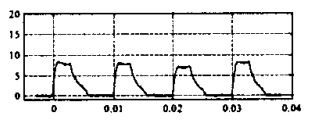

(ii) $\frac{5}{7}$

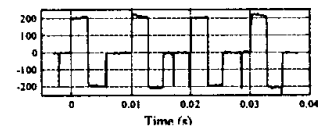

(c)

(i) 长

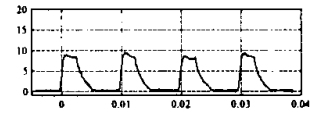

(ii)

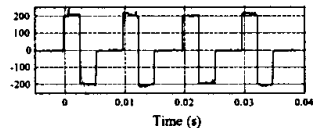

Fig. 7 (i) Phase current and (ii) phase voltages: (a) Simulation results, (b) Experimental result with position sensor and (c) Experimental results without position sensor.

$[\mathrm{Vph}=217 \mathrm{~V}$, Speed $=1000 \mathrm{rpm}$, Load $=6.5 \mathrm{Nm}$.

(a)

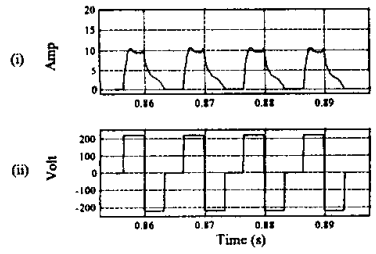

(b)

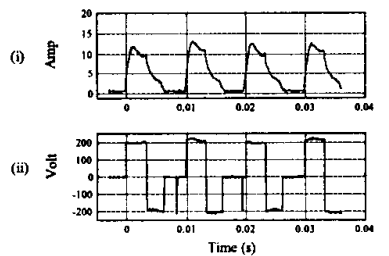

(c)

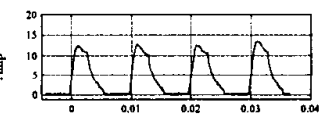

(ii)

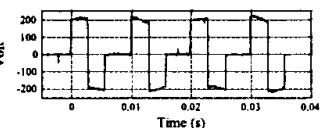

Fig. 8 (i) Phase current and (ii) phase voltages: (a) Simulation results, (b) Experimental result with position sensor and (c) Experimental results without position sensor

$[\mathrm{Vph}=217 \mathrm{~V}$, Speed $=1000 \mathrm{rpm}$, Load $=13 \mathrm{Nm}$.

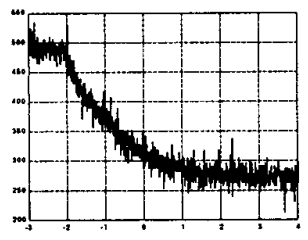

(i)

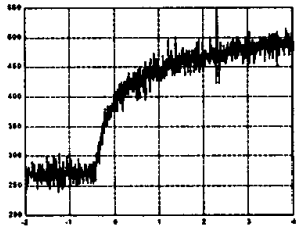

(ii)

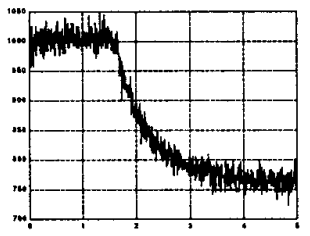

(iii)

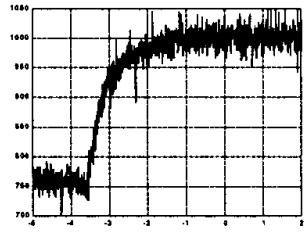

(iv)

Fig. 9 Speed Transients: (i) $500 \mathrm{rpm}$ to $250 \mathrm{rpm}$, (ii) $250 \mathrm{rpm}$ to $500 \mathrm{rpm}$, (iii) $1000 \mathrm{rpm}$ to $750 \mathrm{rpm}$, (iv) $750 \mathrm{rpm}$ to $1000 \mathrm{rpm}$ Horizontal Axes Represent Time in Seconds; Vertical Axes Represent Speed in rpm.

condition of the motor. The highest noise levels reached by the OULTON drive (A), conventional controller (B) and the proposed scheme (C), at different speeds (from $150 \mathrm{rpm}$ to $1500 \mathrm{rpm}$ at the step of $100 \mathrm{rpm}$ ) are shown in Fig. 10 . Figure 11 shows the difference of noise level between our proposed scheme and other conventional schemes. The results clearly show that the proposed method is superior to the conventional control method at low and medium speeds. At high speeds (near the rated speed), however, the difference is not significant as the supply voltage and the 


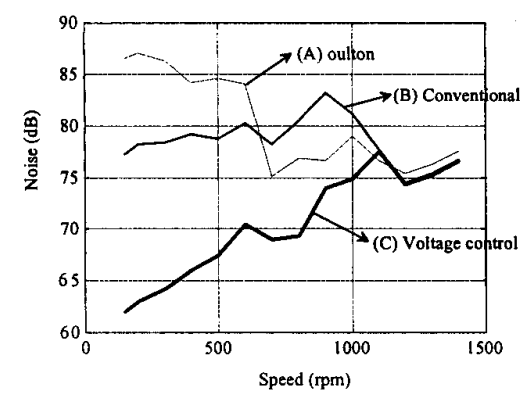

Fig. 10 Maximum Noise level (with background noise of $60 \mathrm{~dB}$ ) at different speed for three cases (A) With OULTON drive,

(B) With DSP controller and Conventional control method, (C) With $\mathrm{DC}$ bus voltage control method

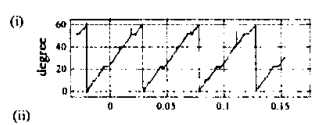

(ii)
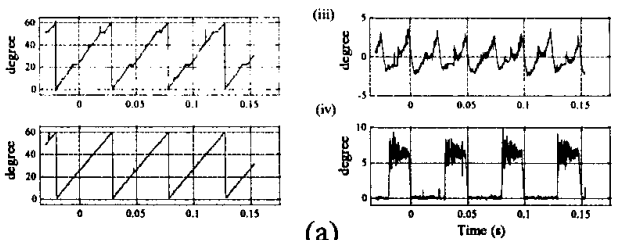

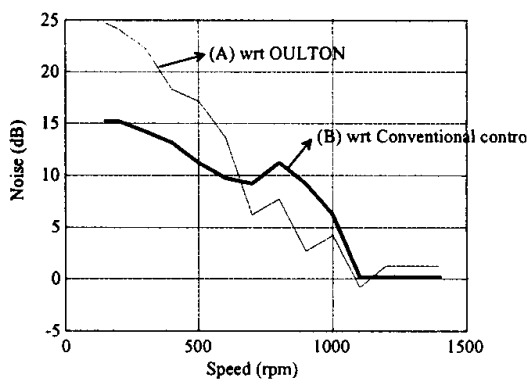

Fig. 11 Improvement in Noise level with dc bus voltage control method at different speed compared to -

(A) OULTON drive (GTO based and with switching frequency of $500 \mathrm{~Hz}$ ), (B) DSP based Conventional control method (with $5 \mathrm{kHz}$ s witching frequency)
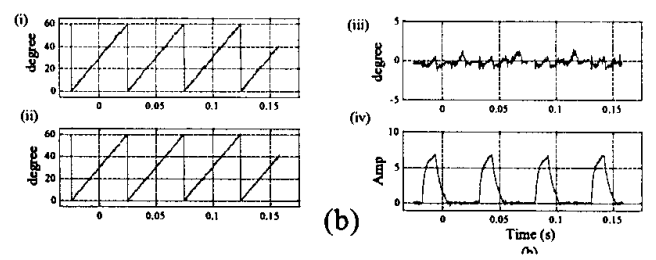

Fig. 12 Test results of (i) Estimated position, (ii) Actual position, (iii) Net error in estimation and (iii) Phase current at 200 rpm in two cases* (a) With Conventional control and $V \mathrm{ph}=220 \mathrm{~V}$, (b) With dc bus voltage control and $\mathrm{Vph}=55 \mathrm{~V}$; $*\left[\right.$ Load $=5 \mathrm{Nm}$, Estimation region $7.5^{\circ}-22.5^{\circ}$; Trace (iii) and (iv) are not synchronised with Trace(i) and (ii)]
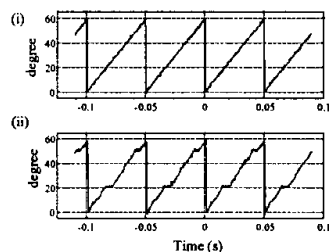

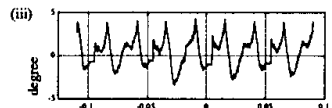

(iv)

(a)

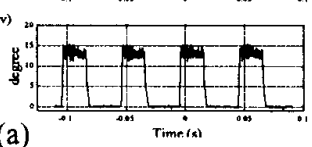

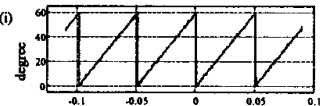

(ii)

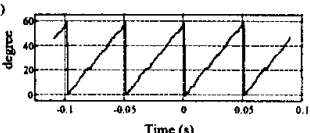

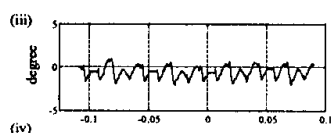

(iv)

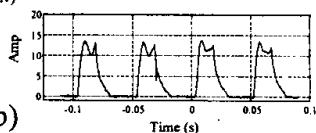

Fig. 13 Test results of (i) Actual position, (ii) Estimated position, (iii) Net error in estimation and (iii) Phase current at 200 rpm in two cases* . (a) With Conventional control and $\mathrm{Vph}=220 \mathrm{~V}$, (b) With dc bus voltage control and $\mathrm{Vph}=55 \mathrm{~V}$;

${ }^{*}\left[\right.$ Load $=18 \mathrm{Nm}$, Estimation region $7.5^{\circ}-22.5^{\circ}$; Trace (iii) and (iv) are not synchronised with Trace(i) and (ii) $]$.

conduction angle are the same. At low speed, a difference in noise level between our conventional control scheme and OULTON drive is observed (c.f. Fig. 11). In case of OULTON drive (GTO based), the switching frequency is only around $500 \mathrm{~Hz}$, whereas in our DSP based controller, the average switching frequency is around $5 \mathrm{kHz}$ (with maximum switching frequency of $10 \mathrm{kHz}$ ). Besides, the $\mathrm{T}$-on, T-off angles are also not the same in both these cases. Thus the difference in noise level in OULTON drive and our conventional control strategy may be attributed to the above causes.

\section{Accurate position estimation}

Since our proposal needs to do away with sensors for estimating rotor positions, the degree of accuracy of its estimate ought to be rigorously tested. The estimated position is compared with the actual position obtained from a known position sensor and the difference between them are plotted as error in estimation. A comparison of performance of the estimator comprising with our conventional DSP controller and the proposed controller is given in Figs 12 and 13. The test results show that the error in estimation with the proposed dc bus voltage control method is always within $\pm 1.5^{\circ}$ irrespective of speed. On the other hand, the error in estimation with conventional controller and at low speeds is as high as $\pm 5^{\circ}$. It may also be seen that for same load and speed, the rms phase current with proposed controller are less than with conventional controller. Thus the torque per ampere of the machine is improved following the proposed method. This improvement in torque per ampere is attributed to the improved accuracy of estimated position. The torque produced by the machine is largely dependent on $\mathrm{T}$-on and $T$-off angles. The accuracy of these angles are dependent on the accuracy of the position estimator. Since, the accuracy of estimator is better with proposed method, the performance of the drive has also improved. Besides, at lower load the conduction angle is less in single pulse mode 
compared to fixed voltage chopping mode (c.f. Fig. 12). The small conduction period reduces the flux integration time which in turn helps improving the accuracy of position estimation. Thus the error due to resistance variation effect is less with single pulse operation compared to fixed voltage chopping mode.

The transient speed responses are shown in Fig. 9. It is seen that the response time with variable dc bus voltage is poor compared to the fixed voltage control method. This can be treated as a trade-off between acoustic noise and response time. By applying rated voltage at low speeds, the dynamic response will improve, but the noise level will increase. Hence, before designing the controller, some compromise has to be made between these two requirements.

\section{ConClusion}

In this paper, an integrated control scheme for SR motor is proposed. The proposed method reduces the noise, and functions without a position or speed sensor. The achievement through the proposed method in noise reduction and accuracy of position estimation is encouraging. The present scheme can be utilised in any variable speed application where acoustics noise specification is stringent. The acoustic noise following the proposed method is compared with the conventional controllers. A significant improvement in noise level is observed with the proposed method compared to the later. A comparison of estimated position with the proposed controller and a conventional controller is made. Unlike, fixed voltage control method, the accuracy of estimation through the proposed method is uniformly good for wide speed range and the error remains within $\pm 1.5^{\circ}$. It is shown that the improved accuracy has effectively improved the torque per ampere rating of the drive.

\section{REFERENCE}

1 D. E. Cameron, J. H. Lang, S. D. Umans, "The Origin of Acoustic Noise in Variable Reluctance Motors", IEEE, IAS Conference Proceedings, pp 108-115, 1989.

2 D. E. Cameron, J. H. Lang, S. D. Umans, "The Origin and Reduction of Acoustic Noise in Doubly-salient Variable-Reluctance Motors", IEEE Transaction on Industiry Applications, Vol. 28, No. 6, November/December, 1992, pp 1250-1255.

$3 \mathrm{C} . \mathrm{Y}$. Wu and Charles Pollock, "Analysis and Reduction of Vibration and Acoustic Noise in the Switched Reluctance Drive", IEEE Transaction on Industry Applications, Vol. 31, No. 1, January/February 1995, pp 91-98.

4 R. S. Colby, F. Mottier, T. J. E. Miller, "Vibration Modes and Acoustic Noise in a 4-phase Switched Reluctance Motor", IEEE, IAS Conference Proceedings, pp 441-447, 1995.

5 M. Besbes, C. Picod, F. Camus, M. Gabsi, "Influence of stator geometry upon vibratory behaviour and electromagnetic performances of switched reluctance motors", IEE Proceedings Power Application, Vol. 145, No. 5, September 1998.

6 Jeff Mahn, D. Williams, Pete Wung, G. Horst, Jerry Lioyd, Steve Randall, "A Systematic Approach Toward Studying Noise and Vibration in Switched Reluctance Machines: Preliminary Results", IEEE, IAS Conference Proceedings, pp 779-785, 1996.

7 C. Pollock and C. Y. Wu, "Acoustic Noise Cancellation Techniques for Switched Reluctance Drives", IEEE, IAS Conference Proceedings, pp 448-455, 1995.

8 A. Michaelides and C. Pollock, "Reduction of Noise and Vibration in Switched Reluctance Motors: New Aspects", IEEE IAS Annual Meeting, 1996, pp $771-778$.

9 W.F. Ray, I.H. Bahadly, "Sensorless Methods for Determining the Rotor Position of Switched Reluctance Motor", Proceedings of 5th European Conference on Power electronics and Applications, IEE publication no. 377, Sept. 13-16, 1993, Vol. 6, pp $7-13$.

10Tom Perl, Iqbal Hussain and Malik Elbuluk, "Design Trends and Trade-offs for Sensorless Operation of Switched Reluctance Motor Drives", IEEE IAS Annual Meeting, 1995, pp 278-285.

11 J. P. Lyons, S. R. Macmin, M. A. Preston, "Flux/Current Method for SRM rotor position estimation", IEEE-Industry Application Society Annual Meeting, 1991, Vol.1, pp. 482-487.

12Debiprasad Panda and V. Ramanarayanan, "An Accurate Position Estimation Method for Switched Reluctance Motor Drive", Published in the Proceedings of International Conference on Power Electronics Drives and Energy Systems (PEDES'98), Perth, Australia, December, 1998, pp 523-528.

13Debiprasad Panda and V. Ramanarayanan, "Mutual Inductance and its Effect on Steady-state performance and Position Estimation Method of Switched Reluctance Motor Drive", IEEE, IAS annual meeting, October 1999, Phoenix, USA.

14Debiprasad Panda and V. Ramanarayanan, "Low noise Switched Reluctance Drive", Published in the Proceedings of PEDES'98, Perth, Australia, December, 1998, pp 523-528.

15 Miller T.J.E.,"Switched Reluctance Motors and Their Control", Magna Physics Publishing And Clarendon Press Oxford 1993. 